\title{
Insulin Need in Gestational Diabetes is Associated with a Worse Cardiovascular Risk Profile after Pregnancy
}

\author{
Firat Bayraktar ${ }^{1}$, Baris Akinci ${ }^{1,2}$, Aygul Celtik ${ }^{3}$, Sunay Tunali ${ }^{4}$, Sinan Genc ${ }^{5}$, \\ Mehmet Ali Ozcan ${ }^{4}$, Mustafa Secil ${ }^{5}$ and Sena Yesil ${ }^{1}$
}

\begin{abstract}
Objective Women with gestational diabetes mellitus (GDM) treated with insulin are more likely to develop type 2 diabetes after pregnancy compared to mild GDM cases treated with medical nutrition treatment (MNT) alone. We aimed to compare levels of subclinical atherosclerosis markers in women with previous GDM treated with insulin and MNT alone.

Methods Eighty-one women with previous GDM (45 treated with insulin, 36 treated with MNT) and 35 age-matched lean controls were included. Fasting glucose, insulin and lipids, circulating fibrinogen, CRP, PAI-1 and IL-6 levels were assayed. Carotid intima media thickness (IMT) was measured.

Results Women with previous GDM treated with insulin in pregnancy had significantly higher fasting glucose, plasma PAI-1 levels and carotid IMT compared to women treated with MNT alone. In multiple regression analysis, insulin need in pregnancy was associated with increased carotid IMT and plasma PAI-1 levels (corrected for age, BMI, postpartum duration, fasting glucose and lipids; model $\mathrm{r}^{2}=0.132$; beta $=0.297, \mathrm{p}=$ 0.014 for carotid IMT; model $\mathrm{r}^{2}=0.198$; beta $=0.345, \mathrm{p}=0.003$ for PAI- 1 ).

Conclusion Women with previous GDM treated with insulin in pregnancy had a worse cardiovascular risk profile compared to mild GDM patients. An intensive preventive approach for cardiovascular disorders is particularly essential for this subgroup of women.
\end{abstract}

Key words: atherosclerosis, carotid IMT, gestational diabetes, subclinical inflammation

(Intern Med 51: 839-843, 2012)

(DOI: 10.2169/internalmedicine.51.5846)

\section{Introduction}

Gestational diabetes mellitus (GDM) is associated with an increased risk of complications for both the mother and fetus. Women with GDM have a higher incidence of preeclampsia and Caesarean section. As a result of excess glucose to be carried via the placenta to the fetus, the fetal pancreas produces extra insulin to regulate blood glucose which leads to macrosomia. Babies born to mothers with GDM are also at increased risk for neonatal hypoglycemia and hyperbilirubinemia $(1,2)$. In addition, women with previous GDM are at an increased risk of developing type 2 diabetes mellitus $(3,4)$, cardiovascular disorders, hyperten- sion, dyslipidemia and metabolic syndrome (4-8).

Optimal glucose control in GDM reduces the risk of developing fetal and maternal complications (2). While medical nutrition is efficient to control blood glucose in a subgroup of women with mild GDM, others need insulin to achieve good glycemic control. It has been shown that women needing additional interventions such as insulin treatment beyond medical nutrition in the course of GDM are more likely to develop type 2 diabetes later $(9,10)$. In this cross-sectional study, we sought to determine the effect of insulin need in GDM on further cardiovascular risk profile after pregnancy.

\footnotetext{
${ }^{1}$ Division of Endocrinology and Metabolism, Department of Internal Medicine, Dokuz Eylul University, Turkey, ${ }^{2}$ Tepecik Research and Training Hospital, Turkey, ${ }^{3}$ Department of Internal Medicine, Dokuz Eylul University, Turkey, ${ }^{4}$ Division of Hematology, Department of Internal Medicine, Dokuz Eylul University, Turkey and ${ }^{5}$ Department of Radiology, Dokuz Eylul University, Turkey

Received for publication May 19, 2011; Accepted for publication December 19, 2011

Correspondence to Dr. Baris Akinci, barisakincimd@gmail.com
} 


\section{Materials and Methods}

Ninety consecutive women with previous GDM were screened. After exclusion of 9 women who had newly diagnosed diabetes or hypertension, 81 women were included. The control group consisted of 35 lean women with a normal screening for GDM at the same period as the study group. Women with known arterial or venous disease, hypertension, familial hyperlipidemia, chronic inflammatory disease, coagulation disorders and other systemic diseases and postmenopausal women were not included. Smokers were excluded. GDM had been diagnosed by screening with a 50-g 1-h glucose challenge test at 24-28th weeks of gestation, followed by a 100-g 3-h oral glucose tolerance test (OGTT) at Dokuz Eylul University Hospital between January 2002 and January 2008. Women with severe obesity, prior history of GDM or delivery of large-for-gestationalage-infant, diagnosis of polycystic ovary syndrome, and strong family history for type 2 diabetes had been screened at their initial prenatal visits because of the high risk for developing GDM. Revised Carpenter and Coustan criteria had been used for the GDM diagnosis (11).

In pregnancy complicated with GDM, medical nutrition treatment (MNT) had been prescribed by a registered dietician. MNT had been individualized in respect to prepregnancy BMI, weight gain during pregnancy, physical activity and cultural characteristics of each patient. Patients had been trained in carbohydrate counting, use of food records, and self-monitoring to facilitate the glycemic control. Physical activity for 30 minutes per day had been recommended to all patients. Patients had been instructed to monitor their blood glucose levels. Target maternal capillary glucose levels had been $<96 \mathrm{mg} / \mathrm{dL}$ at fasting, $<140 \mathrm{mg} / \mathrm{dL}$ at 1 hour, and $<120 \mathrm{mg} / \mathrm{dL}$ at 2 hours after starting the meal, according to Fifth International Workshop Conference on GDM (12). Patients who had not achieved target levels had been treated with insulin. Human regular and NPH insulin preparations had been used in multiple injection regimens. The insulin dose was adjusted accordingly on the basis of self-obtained capillary glucose levels. None of the patients had received long or rapid acting insulin analogs. None of the patients had been treated with oral antihyperglycemic agents.

Height, weight and waist circumference were measured. Body mass index (BMI) was calculated. Blood pressure was measured using a sphygmomanometer in the sitting position after 5 minutes rest. Subjects with a blood pressure level higher than $130 \mathrm{mmHg}$ for systolic and $80 \mathrm{mmHg}$ for diastolic at the examination or patients using antihypertensive drugs were defined as hypertensive and excluded.

Blood was taken from the cannulated antecubital vein between 8:00 AM and 9:00 AM after 10-hour overnight fasting. In order to evaluate carbohydrate intolerance, 75 -g oral glucose tolerance test (OGTT) was performed. Women with type 2 diabetes, which was defined according to American
Diabetes Association (ADA) recommendations (11), were excluded. Fasting insulin levels were assayed, and homeostasis model assessment (HOMA) score was calculated. Circulating lipids, fibrinogen, high sensitive CRP and IL-6 levels were assayed. Glucose levels were measured by a colorimetric method with the Roche/Hitachi D/P Modular System Autoanalyzer (Roche Diagnostics, Basel, Switzerland). Triglycerides, total cholesterol and high density lipoprotein (HDL) cholesterol were measured by Roche/Hitachi D/P Modular System Autoanalyzer (Roche Diagnostics, Basel, Switzerland). Low density lipoprotein (LDL) cholesterol was calculated by the Friedewald's equation method (13). Fibrinogen was measured by the Clauss method (14). Creactive protein (CRP, Biomerica, Newport Beach, CA, USA), interleukin-6 (IL-6, Invitrogen, Grand Island, NY, USA) and plasminogen activator inhibitor-1 (PAI-1, Bender Medical Systems, Vienna, Austria) assays were done using commercial ELISA kits according to manufacturers' introductions.

Carotid intima media thickness (IMT) was measured by a radiologist using a high resolution Doppler ultrasound (Philips HDI 5000, Bothell, WA, USA). Ultrasonographic images of the right and the left common carotid arteries (CCA) of each case at the lower $1 / 3$ cervical region proximally and $1 \mathrm{~cm}$ above the carotid bulb distally in longitudinal plane were obtained. Measurements of the proximal and distal CCA posterior wall were done manually by the provided distance measurement system of the ultrasonography device after magnification of the images. Three measurements were made in a non-neighboring fashion within an approximately $1 \mathrm{~cm}$ segment both from the left and right CCA proximal and distal portions. IMT thickness values were then calculated by obtaining the arithmetic means of the measured values.

Variable distributions were assessed by the KolmogorovSmirnov normality test. Independent sample t-test was used to compare variables of patients. One way ANOVA with Bonferroni correction was applied for comparison of subgroups. Categorical variables were compared by the chisquare test. Correlation analyses were performed using Pearson's coefficients. Regression analysis was employed to assess correlations between studied parameters. Statistical analysis was performed using Statistical Package of Social Science (SPSS Inc, Chicago, IL, USA), version 15.0 for Windows. Data were expressed as mean \pm standard deviation (SD). A p value less than 0.05 was accepted as statistically significant.

\section{Results}

Table 1 summarizes the characteristics of women with previous GDM and age-matched lean controls. As expected, women with previous GDM were more obese. They had elevated fasting glucose levels, HOMA scores and more atherogenic lipid profiles. Women with previous GDM had significantly increased fibrinogen, CRP, PAI-1 and IL-6 levels and 
Table 1. Baseline Characteristics of Women with Previous GDM and Agematched Lean Controls

\begin{tabular}{cccc}
\hline & $\begin{array}{c}\text { Previous GDM } \\
(\mathrm{n}=81)\end{array}$ & $\begin{array}{c}\text { Controls } \\
(\mathrm{n}=35)\end{array}$ & p value \\
\hline Age (years) & $35.07 \pm 5.33$ & $33.6 \pm 5.49$ & 0.178 \\
Postpartum duration (months) & $3.11 \pm 1.76$ & $3.23 \pm 1.56$ & 0.734 \\
BMI $\left(\mathrm{kg} / \mathrm{m}^{2}\right)$ & $27.55 \pm 4.38$ & $22.64 \pm 2.06$ & $<0.001$ \\
Waist $(\mathrm{cm})$ & $91.95 \pm 12.18$ & $79.43 \pm 9.32$ & $<0.001$ \\
Fasting glucose $(\mathrm{mg} / \mathrm{dL})$ & $97.41 \pm 12.68$ & $78.17 \pm 9.8$ & $<0.001$ \\
Total cholesterol $(\mathrm{mg} / \mathrm{dL})$ & $197.06 \pm 35.62$ & $172.03 \pm 30.85$ & $<0.001$ \\
Triglyceride $(\mathrm{mg} / \mathrm{dL})$ & $112.75 \pm 49.37$ & $83.63 \pm 37.98$ & 0.002 \\
HDL cholesterol $(\mathrm{mg} / \mathrm{dL})$ & $51.48 \pm 12.01$ & $60.68 \pm 15.03$ & 0.001 \\
LDL cholesterol $(\mathrm{mg} / \mathrm{dL})$ & $123.03 \pm 31.06$ & $94.62 \pm 26.51$ & $<0.001$ \\
Fibrinogen $(\mathrm{g} / \mathrm{L})$ & $4.12 \pm 0.99$ & $3.48 \pm 0.62$ & $<0.001$ \\
HOMA & $2.02 \pm 1.56$ & $1.05 \pm 0.95$ & $<0.001$ \\
Carotid IMT (mm) & $0.55 \pm 0.07$ & $0.52 \pm 0.05$ & 0.014 \\
CRP $(\mathrm{mg} / \mathrm{L})$ & $2.57 \pm 2.11$ & $1.31 \pm 1.56$ & 0.001 \\
IL-6 $(\mathrm{pg} / \mathrm{mL})$ & $2.49 \pm 1.2$ & $1.99 \pm 0.85$ & 0.028 \\
PAI-1 (ng/mL) & $124.07 \pm 27.25$ & $108.55 \pm 27.21$ & 0.006 \\
\hline
\end{tabular}

Table 2. Comparison of Women Treated with Insulin and MNT, and Agematched Lean Controls

\begin{tabular}{|c|c|c|c|}
\hline & $\begin{array}{l}\text { Insulin } \\
(\mathrm{n}=45)\end{array}$ & $\begin{array}{c}\text { MNT } \\
(n=36)\end{array}$ & $\begin{array}{l}\text { Controls } \\
(\mathrm{n}=35)\end{array}$ \\
\hline Age (years) & $34.76 \pm 5.32$ & $35.47 \pm 5.37$ & $33.6 \pm 5.49$ \\
\hline Postpartum duration (months) & $2.84 \pm 1.62$ & $3.44 \pm 1.89$ & $3.23 \pm 1.56$ \\
\hline BMI $\left(\mathrm{kg} / \mathrm{m}^{2}\right)^{* \dagger}$ & $27.98 \pm 4.58$ & $27.01 \pm 4.12$ & $22.64 \pm 2.06$ \\
\hline Waist $(\mathrm{cm})^{* \dagger}$ & $93.38 \pm 12.09$ & $90.17 \pm 12.23$ & $79.43 \pm 9.32$ \\
\hline Fasting glucose $(\mathrm{mg} / \mathrm{dL})^{* \dagger} \ddagger$ & $100.56 \pm 4.01$ & $93.47 \pm 9.61$ & $78.17 \pm 9.8$ \\
\hline Total cholesterol $(\mathrm{mg} / \mathrm{dL})^{* \dagger}$ & $199.76 \pm 36.36$ & $193.69 \pm 34.88$ & $172.03 \pm 30.85$ \\
\hline Triglyceride $(\mathrm{mg} / \mathrm{dL})^{*}$ & $120.91 \pm 56.64$ & $102.56 \pm 36.68$ & $83.63 \pm 37.98$ \\
\hline HDL cholesterol $(\mathrm{mg} / \mathrm{dL})^{* \dagger}$ & $51.44 \pm 12.53$ & $51.53 \pm 11.49$ & $60.68 \pm 15.03$ \\
\hline LDL cholesterol (mg/dL) ${ }^{* \dagger}$ & $124.13 \pm 32.89$ & $121.66 \pm 28.99$ & $94.62 \pm 26.51$ \\
\hline Fibrinogen $(\mathrm{g} / \mathrm{L})^{* \dagger}$ & $4.18 \pm 1.06$ & $4.05 \pm 0.92$ & $3.48 \pm 0.62$ \\
\hline HOMA $^{*}$ & $2.19 \pm 1.76$ & $1.79 \pm 1.25$ & $1.05 \pm 0.95$ \\
\hline Carotid IMT $(\mathrm{mm}) * *$ & $0.57 \pm 0.07$ & $0.53 \pm 0.05$ & $0.52 \pm 0.05$ \\
\hline $\mathrm{CRP}(\mathrm{mg} / \mathrm{L})^{* \dagger}$ & $2.66 \pm 1.16$ & $2.46 \pm 2.09$ & $1.31 \pm 1.56$ \\
\hline $\mathrm{IL}-6(\mathrm{pg} / \mathrm{mL})^{*}$ & $2.74 \pm 1.4$ & $2.19 \pm 0.81$ & $1.99 \pm 0.85$ \\
\hline PAI-1 (ng/mL) ${ }^{*}$ & $132.87 \pm 26.76$ & $113.06 \pm 23.92$ & $108.55 \pm 27.21$ \\
\hline
\end{tabular}

Table 3. Correlation Coefficients between Carotid IMT and Studied Parameters

\begin{tabular}{ccc}
\hline & $\mathrm{r}$ & $\mathrm{p}$ \\
\hline BMI & 0.237 & 0.01 \\
Fasting glucose & 0.234 & 0.011 \\
CRP & 0.2 & 0.031 \\
IL-6 & 0.229 & 0.013 \\
PAI-1 & 0.386 & $<0.001$ \\
\hline
\end{tabular}

carotid IMT measurements.

Table 2 shows the comparison of women treated with insulin and MNT alone, and age-matched lean controls. Women with previous GDM treated with insulin were more obese $(\mathrm{p}<0.001$ for BMI and waist), had elevated total $(\mathrm{p}=$ $0.002)$ and LDL $(\mathrm{p}<0.001)$ cholesterol, triglyceride $(\mathrm{p}=$ $0.001)$, fasting glucose $(\mathrm{p}<0.001)$, HOMA $(\mathrm{p}=0.001)$, fibrinogen $(p=0.002)$, CRP $(p=0.009)$, IL-6 ( $p=0.009)$, PAI-1 $(\mathrm{p}<0.001)$, carotid IMT $(0.001)$ and decreased HDL cholesterol $(\mathrm{p}=0.006)$ levels compared to controls. Women with previous GDM treated with MNT alone were also more obese when compared with controls $(\mathrm{p}<0.001$ for BMI and waist). They had elevated total $(\mathrm{p}=0.027)$ and LDL cholesterol $(\mathrm{p}=0.001)$, fasting glucose $(\mathrm{p}<0.001)$, fibrinogen $(\mathrm{p}=$ $0.025)$, CRP $(p=0.048)$ and decreased HDL cholesterol levels $(\mathrm{p}=0.011)$. Women with previous GDM treated with insulin in pregnancy had significantly higher fasting glucose $(\mathrm{p}=0.022)$, plasma PAI-1 levels $(\mathrm{p}=0.03)$ and carotid IMT measurements $(\mathrm{p}=0.007)$ compared to women treated with MNT alone.

Carotid IMT was positively correlated with BMI, fasting glucose, CRP, PAI-1 and IL-6 (Table 3). In multiple regression analysis, insulin need in pregnancy complicated with GDM was independently associated with carotid IMT and plasma PAI-1 (corrected for age, BMI, postpartum duration, fasting glucose and lipid levels; model $\mathrm{r}^{2}=0.132$; beta $=0.297$, $\mathrm{p}=0.014$ for carotid IMT; model $\mathrm{r}^{2}=0.198$; beta $=0.345, \mathrm{p}=$ 0.003 for PAI-1).

\section{Discussion}

Traditional risk factors for atherosclerosis include age, gender, family history, smoking, being overweight or obese, 
sedentary life style, diabetes, hypertension and hyperlipidemia. Non-pharmaceutical means are essential in the management of atherosclerosis, such as cessation of smoking, practicing regular exercise and nutritional modifications. A pharmacological approach, which targets the elimination of every modifiable risk factor, is generally the next step in preventing atherosclerosis. Endothelial dysfunction is the initiating factor that causes atherosclerosis. Circulating levels of inflammatory factors and fibrinolytic system proteins have been associated with endothelial dysfunction and atherosclerosis (15). In this respect, the assessment of circulating markers of endothelial dysfunction, insulin resistance and the measurement of carotid IMT are a non-invasive way to assess the degree of subclinical atherosclerosis. Insulin resistance can be investigated by various methods such as insulin sensitivity index, HOMA, hyperinsulinemic euglycemic clamp, and the assessment of associated circulating markers such as leptin, adiponectin, PAI-1, IL-6 and TNF- $\alpha$ as well (16). In accordance with previous studies, the present data suggested that women with previous GDM had an increased cardiovascular risk after pregnancy. Beyond that, we found that women treated with insulin in their pregnancy complicated with GDM had elevated levels of subclinical atherosclerosis markers compared to those treated with MNT alone.

Previous studies have reported that the rate of developing diabetes after index pregnancy complicated with GDM is increased in women treated with insulin $(4,9,10)$. It is well known that women with GDM have both defects in insulin sensitivity and insulin secretion as in type 2 diabetes (17). Insulin need in the course of GDM is a marker of relatively reduced endogenous insulin secretion capacity. Thus, insulin need during pregnancy may be considered as an indicator for the failure of pancreatic beta cell function and it is plausible that this group of women more likely develop diabetes later. In the present study, we did not include women developing type 2 diabetes. However, fasting glucose levels were still higher in women treated with insulin. Therefore, we propose that women with GDM who need insulin in pregnancy are candidates for developing type 2 diabetes after pregnancy, as has been previously shown in non-diabetic people with glucose intolerance (18-20); they may develop the signs of subclinical atherosclerosis before developing type 2 diabetes.

In a very recent study that recruited approximately 25,000 pregnant women at 15 centers in nine countries, the Hyperglycemia and Adverse Pregnancy Outcome (HAPO) study (21), a strong linear association was found between higher levels of maternal plasma glucose and macrosomia, an association that remained significant after making adjustments for field center, maternal body mass index, height, or age. The study showed that the risk of adverse maternal, fetal, and neonatal outcomes continuously increased as a consequence of maternal hyperglycemia, even within the ranges previously considered normal for pregnancy. Reconsidering these important results from the HAPO study, the Interna- tional Association of Diabetes and Pregnancy Study Groups (IADPSG) developed more strict recommendations for diagnosing GDM (22). Based on the evidence from the Hyperglycemia and Adverse Pregnancy Outcomes (HAPO) study and recommendations of the IADPSG, most organizations from different countries including Japan have adopted the recommendations into their standards of care. This new set of recommendations for screening and diagnosis of GDM has also been widely confirmed by the American Diabetes Association (ADA) in the standards of medical care in diabetes-2011 article (23). According to these newly developed diagnostic cut off points for the fasting, 1-h, and 2-h plasma glucose measurements, it is very likely that the prevalence of GDM will be significantly increased, in particular mild GDM which is usually treated with medical nutrition and lifestyle therapy alone. Although physicians around the world from different specialties dealing with GDM have long recognized a need for more consensus on diagnosis and screening for gestational diabetes, it should be noted that not all medical associations, including the American Congress of Obstetricians and Gynecologists (ACOG), have accepted the international guidelines. Some researchers are still concerned that the new screening process, which could raise the prevalence of GDM, may be too demanding. Beyond plausible concerns about the medicalization of pregnancies previously categorized as normal, this new approach will be associated with increased cost. The increased cost will be due in part to more intensive follow-up of mothers during pregnancy. The new international guidelines on screening for GDM that will likely raise the prevalence of the condition have sparked questions over the appropriate diagnostic tools and cost efficacy also in Turkey. The Society of Endocrinology and Metabolism of Turkey has not yet made any suggestion for the use of the IADPSG recommendations countrywide. In view of these concerns, instead, the Turkish Society recommends physicians to feel free to use both diagnostics approaches until more data on the benefits and cost-efficacy of the IADPSG recommendations are collected. On the other hand, with the potential increase in the prevalence of mild GDM cases in the future, efforts should be maintained for determining high risk subjects for cardiovascular risk among women with previous GDM. The present study constitutes a good example for such efforts. Our results reveal that insulin need in index pregnancy complicated with GDM is associated with an increased further cardiovascular risk. Thus, meticulous care should be established for this subgroup of women with previous GDM to prevent cardiovascular disorders.

There were some limitations of our study. First, GDM was diagnosed using previous recommendations of ADA (Carpenter and Coustan criteria), as our study was designed before the IADPSG released the new set of recommendations for the diagnosis of GDM. Second, it is also necessary to consider the effects of ethnic differences on the diagnosis and course of GDM. Further studies investigating the cardiovascular risk in women with previous GDM from differ- 
ent ethnic groups who were previously categorized as normal are necessary. Third, since the control group was generated from lean subjects, the BMI values of GDM and control groups were statistically different in our study. As GDM has been clearly associated with obesity, it was problematic to match both groups for BMI. This could be achieved by including some overweight women without previous GDM in the study protocol. However, in that way, the control group would consist of mostly overweight women. Previous studies have already shown that the measures of atherosclerosis are different in women with previous GDM. In the current study, our main goal was to determine the effect of insulin need in pregnancy on the cardiovascular risk profile. As shown in Table 2, there was no statistical difference in BMI between previous GDM women treated with insulin and MNT alone. Furthermore, insulin need in pregnancy complicated with GDM was independently associated with carotid IMT and plasma PAI-1 when the data was corrected for age, BMI, postpartum duration, fasting glucose and lipid levels.

In conclusion, we suggest that meticulous strategies should be developed to prevent cardiovascular disorders in women with previous GDM. For this purpose, the determination of women who are at very high risk for developing cardiovascular disorders might help clinicians in planning the management strategies. Women with GDM needing insulin to control their blood glucose have a worse cardiovascular profile after pregnancy. An intensive preventive approach including lifestyle modification, prevention of obesity, exercise and intensive management of glucose intolerance, hypertension and dyslipidemia seems to be in particular essential for this subgroup of women.

The authors state that they have no Conflict of Interest (COI).

\section{References}

1. Reece EA, Leguizamon G, Wiznitzer A. Gestational diabetes: the need for a common ground. Lancet 373: 1789-1797, 2009.

2. Dennedy MC, Dunne F. The maternal and fetal impacts of obesity and gestational diabetes on pregnancy outcome. Best Pract Res Clin Endocrinol Metab 24: 573-589, 2010.

3. Akinci B, Celtik A, Yener S, Yesil S. Is fasting glucose level during oral glucose tolerance test an indicator of the insulin need in gestational diabetes? Diabetes Res Clin Pract 82: 219-225, 2008.

4. Akinci B, Celtik A, Genc S, et al. Evaluation of postpartum carbohydrate intolerance and cardiovascular risk factors in women with gestational diabetes. Gynecol Endocrinol 27: 361-367, 2011.

5. Akinci B, Celtik A, Yener S, Yesil S. Prediction of developing metabolic syndrome after gestational diabetes mellitus. Fertil Steril 93: 1248-1254, 2010.

6. Akinci B, Demir T, Celtik A, et al. Serum osteoprotegerin is associated with carotid intima media thickness in women with previous gestational diabetes. Diabetes Res Clin Pract 82: 172-178, 2008.

7. Akinci B, Celtik A, Yuksel F, et al. Increased osteoprotegerin levels in women with previous gestational diabetes developing metabolic syndrome. Diabetes Res Clin Prac 91: 26-31, 2011.

8. Bentley-Lewis R. Late cardiovascular consequences of gestational diabetes mellitus. Semin Reprod Med 27: 322-329, 2009.

9. Kjos SL, Peters RK, Xiang A, et al. Predicting future diabetes in Latino women with gestational diabetes. Utility of early postpartum glucose tolerance testing. Diabetes 44: 586-591, 1995.

10. Madarasz E, Tamas G, Tabak AG, Kerenyi Z. Carbohydrate metabolism and cardiovascular risk factors 4 years after a pregnancy complicated by gestational diabetes. Diabetes Res Clin Pract 85: 197-202, 2009.

11. Diagnosis and classification of diabetes mellitus. Diabetes Care $\mathbf{3 3}$ Suppl 1: S62-S69, 2010.

12. Metzger BE, Buchanan TA, Coustan DR, et al. Summary and recommendations of the Fifth International Workshop-Conference on Gestational Diabetes Mellitus. Diabetes Care 30 Suppl 2: S251S260, 2007.

13. Friedewald WT, Levy RI, Fredrickson DS. Estimation of the concentration of low-density lipoprotein cholesterol in plasma, without use of the preparative ultracentrifuge. Clin Chem 18: 499-502, 1972.

14. Clauss A. Rapid physiological coagulation method in determination of fibrinogen. Acta Haematol 17: 237-246, 1957.

15. Brandenburg SL, Lindenfeld J, Reusch JE, Regensteiner JG. Cardiovascular risk in women with type 2 diabetes. Med Clin North Am 87: 955-969, 2003.

16. Frishman WH. Biologic markers as predictors of cardiovascular disease. Am J Med 1998 104: 18S-27S, 1998.

17. Catalano PM. Carbohydrate metabolism and gestational diabetes. Clin Obstet Gynecol 37: 25-38, 1994.

18. Ford ES, Zhao G, Li C. Pre-diabetes and the risk for cardiovascular disease: a systematic review of the evidence. J Am Coll Cardiol 55: 1310-1317, 2010.

19. Anselmino M, Wallander M, Norhammar A, Mellbin L, Ryden L. Implications of abnormal glucose metabolism in patients with coronary artery disease. Diab Vasc Dis Res 5: 285-290, 2008.

20. Hsueh WA, Orloski L, Wyne K. Prediabetes: the importance of early identification and intervention. Postgrad Med 122: 129-143, 2010.

21. Hyperglycemia and Adverse Pregnancy Outcome (HAPO) Study: associations with neonatal anthropometrics. Diabetes 58: 453-459, 2009.

22. Metzger BE, Gabbe SG, Persson B, et al. International association of diabetes and pregnancy study groups recommendations on the diagnosis and classification of hyperglycemia in pregnancy. Diabetes Care 33: 676-682, 2010.

23. Standards of medical care in diabetes--2011. Diabetes Care 34 Suppl 1: S11-S61, 2011

(C) 2012 The Japanese Society of Internal Medicine

http://www.naika.or.jp/imindex.html 